DESY 09-134, TUM-HEP 736/09

\title{
Detecting Gamma-Ray Anisotropies from Decaying Dark Matter: Prospects for Fermi LAT
}

\author{
Alejandro Ibarre* and David Tran \\ Physik-Department T30d, Technische Universität München, \\ James-Franck-Straße, 85748 Garching, Germany. \\ Christoph Weniger \\ Deutsches Elektronen-Synchrotron DESY, \\ Notkestraße 85, 22607 Hamburg, Germany.
}

\begin{abstract}
Decaying dark matter particles could be indirectly detected as an excess over a simple power law in the energy spectrum of the diffuse extragalactic gamma-ray background. Furthermore, since the Earth is not located at the center of the Galactic dark matter halo, the exotic contribution from dark matter decay to the diffuse gamma-ray flux is expected to be anisotropic, offering a complementary method for the indirect search for decaying dark matter particles. In this paper we discuss in detail the expected dipole-like anisotropies in the dark matter signal, taking also into account the radiation from inverse Compton scattering of electrons and positrons from dark matter decay. A different source for anisotropies in the gamma-ray flux are the dark matter density fluctuations on cosmic scales. We calculate the corresponding angular power spectrum of the gamma-ray flux and comment on observational prospects. Finally, we calculate the expected anisotropies for the decaying dark matter scenarios that can reproduce the electron/positron excesses reported by PAMELA and the Fermi LAT, and we estimate the prospects for detecting the predicted gamma-ray anisotropy in the near future.
\end{abstract}

\section{INTRODUCTION}

The full-sky observations of gamma rays undertaken in the 1990s by the Energetic Gamma Ray Experiment Telescope (EGRET) revealed a map which is highly anisotropic. It showed a number of resolved sources [1], such as blazars, as well as an unresolved component, which can be attributed almost entirely to Galactic emission. The mechanisms which produce the diffuse Galactic emission (inverse Compton scattering, $\pi^{0}$ production and bremsstrahlung) are well understood. However, the actual intensity of each of these contributions depends on many details of the propagation of cosmic rays in the Galaxy, including the interstellar radiation and magnetic fields and the Galactic gas distribution. On the other hand, over the last years a picture of cosmic-ray propagation has emerged which can account for the observed abundances of almost all secondary cosmic-ray species. Remarkably, this same propagation model can reproduce, using the interstellar gas and radiation field distributions and a Galactic magnetic field inferred from observations, the full-sky gamma-ray map with rather good accuracy. However, this requires the introduction of an additional, a priori undetermined, diffuse component which is postulated to be of extragalactic origin and thus isotropic. Its energy spectrum is determined by observations at high Galactic latitudes.

Recently it has become apparent that the state-of-the-

\footnotetext{
*Electronic address: alejandro.ibarra@ph.tum.de

${ }^{\dagger}$ Electronic address: david.tran@ph.tum.de

${ }^{\ddagger}$ Electronic address: christoph.weniger@desy.de
}

art propagation models fail to reproduce the measurements of the positron fraction at energies larger than $10 \mathrm{GeV}$. Namely, the secondary positron flux calculated with current propagation models, together with the total electron-plus-positron spectrum measured by the Fermi LAT, yields a positron fraction which monotonically decreases with energy, whereas the PAMELA observations reveal that above $10 \mathrm{GeV}$ the positron fraction increases with energy [2, 3, 4]. This puzzling behavior, already suggested in the past by a series of experiments such as HEAT [5], has been interpreted as evidence for a primary component of the positron flux (possibly accompanied by an identical electron flux). Furthermore, the conventional propagation model adopted by the Fermi collaboration, dubbed "model 0", which fits well the low-energy data points of the total electron-plus-positron flux and the positron fraction, fails to reproduce the observations at higher energies, reinforcing the necessity of an exotic source of primary electrons and positrons.

The most common astrophysical explanation of the electron/positron excesses is the electron-positron pair production by the interactions of high-energy photons in the strong magnetic field of nearby pulsars, such as Geminga or Monogem [6, 7, 8, 9]. However, this interpretation requires a rather large percentage of the total spin-down power injected in the form of electron-positron pairs, namely about $40 \%$, and a large cutoff of the electron/positron energy spectrum at about $1 \mathrm{TeV}$. Alternatively, the electron/positron excesses could be explained by the combined emission of both nearby and distant pulsars, this solution requiring a percentage of spin-down power ranging between $10-30 \%$ and again a large cutoff in the energy spectrum, $800-1400 \mathrm{GeV}$ [2].

An arguably more exciting explanation of the cosmic- 
ray electron/positron excesses is the possibility that the electrons and positrons are produced in the annihilation [10, 11, 12, 13] or the decay [14, 15, 16, 17, 18] of dark matter particles. Despite the overwhelming gravitational evidence for the existence of non-baryonic dark matter, which makes up more than one-fifth of the energy budget of the Universe [19], very little is known about its particle nature (see Ref. [20] for a review). The most popular type of dark matter candidate, the weakly interacting massive particle (WIMP), can naturally reproduce the observed dark matter abundance due to effective selfannihilation in the early Universe, after being in thermal equilibrium with the baryons before. Today, this same annihilation process is expected to produce a possibly observable contribution to the measured cosmic-ray fluxes on Earth. Detection of such an indirect signal would be the first non-gravitational evidence for dark matter, with paramount importance to the understanding of its nature.

A lot of effort has been made to study the prospects and predictions of cosmic-ray signatures from annihilating dark matter, see e.g. [21, 22, 23]. However, this is not the only possibility for the indirect detection of dark matter. Many dark matter models predict that the dark matter particle is unstable and decays with very long lifetimes $14,17,18,24,25]$. If the decays occur at a sufficiently large rate, the decay products could be observable as an exotic contribution to the high energy cosmic ray fluxes of gamma rays, electrons, positrons, antiprotons, neutrinos or antideuterons [26, 27, 28, 29, 30, 31, 32]. Among these, the gamma-ray channel is probably the most important to study, due to its sensitivity to fardistant sources and its potential to discriminate between signals from annihilating or decaying dark matter and astrophysical sources.

In this paper we will present a detailed study of the peculiar predictions for gamma rays from decaying dark matter. We will concentrate on their angular anisotropies on large and small scales. These can be used to discriminate this component from other contributions to the extragalactic diffuse emission. It turns out that the predictions for decaying dark matter are much more robust than the ones for annihilation, which makes this scenario very predictive and easier to confirm or falsify [33]. Secondly, we will discuss the prospects to see such signals in the upcoming Fermi LAT gamma-ray data, and we will apply our results to the decaying dark matter explanation of the electron/positron excess.

This paper is organized as follows: In section II we will review the basic concepts about production, propagation and absorption of gamma rays from dark matter decay. In section III we will calculate the gamma-ray anisotropy expected from the decay of dark matter particles on large angular scales. We will show in particular the expected anisotropy in scenarios which can explain the electron/positron excesses observed by PAMELA and the Fermi LAT, and we will argue that this anisotropy should be seen by the Fermi LAT. In section IV we will calculate the angular power spectrum of gamma rays from decaying dark matter on small angular scales and comment on its observational prospects. Lastly, in section $\mathrm{V}$, we will present our conclusions. We also present two appendices in which we discuss statistical properties of the large-scale anisotropy and the general observational strategy for gamma rays from dark matter decay.

\section{GAMMA RAYS FROM DARK MATTER DECAY}

The total gamma-ray flux from dark matter decay receives several contributions. The first one stems from the decay of dark matter particles in the Milky Way halo and reads

$$
\frac{d J_{\text {halo }}}{d E_{\gamma}}(l, b)=\frac{1}{4 \pi m_{\mathrm{dm}} \tau_{\mathrm{dm}}} \frac{d N_{\gamma}}{d E_{\gamma}} \int_{0}^{\infty} d s \rho_{\text {halo }}[r(s, l, b)],
$$

where $d N_{\gamma} / d E_{\gamma}$ is the energy spectrum of gamma rays produced in the decay of a dark matter particle and $\rho_{\text {halo }}(r)$ is the density profile of dark matter particles in our Galaxy, as a function of the distance from the Galactic center, $r$. The received gamma-ray flux depends on the Galactic coordinates, longitude $l$ and latitude $b$, and is given by a line-of-sight integral over the parameter $s$, which is related to $r$ by

$$
r(s, l, b)=\sqrt{s^{2}+R_{\odot}^{2}-2 s R_{\odot} \cos b \cos l} .
$$

Here, $R_{\odot}=8.3 \mathrm{kpc}$ denotes the distance of the Sun to the Galactic center 34].

The latest $N$-body simulations favor the Einasto density profile $22,35,36$.

$$
\rho_{\text {halo }}^{\text {Einasto }}(r) \propto \exp \left[-\frac{2}{\alpha}\left(\left(\frac{r}{r_{s}}\right)^{\alpha}-1\right)\right],
$$

which we use throughout the work when not stated otherwise, and for which we adopt $\alpha=0.17$ and the scale radius $r_{s}=20 \mathrm{kpc}$. For comparison we will also show results for the much shallower isothermal profile

$$
\rho_{\text {halo }}^{\text {isothermal }}(r) \propto \frac{1}{r^{2}+r_{s}^{2}}
$$

with $r_{s}=3.5 \mathrm{kpc}$. We use the local dark matter density as determined in Ref. [37], $\rho_{\odot}=0.385 \mathrm{GeV} \mathrm{cm}^{-3}$, to normalize the profiles at the position of the Sun. Related uncertainties and their impact on our results will be discussed below.

The electrons and positrons that can be produced in the decay of dark matter particles, and which may be the origin of the PAMELA and Fermi LAT anomalies, also generate a contribution to the total gamma-ray flux through their inverse Compton scattering on the interstellar radiation field (ISRF), which includes the CMB, 
thermal dust radiation and starlight. Recently, ICS radiation in connection with the PAMELA excess was discussed in Refs. 38, 39, 40, 41, 42, 43]; a pedagogical review can be found in Ref. [44]. Furthermore, the interactions of energetic electrons and positrons with the Galactic magnetic field produce synchrotron radiation in the radio band with frequencies $\mathcal{O}(0.1-100 \mathrm{GHz})$, which could also be observed (see e.g. Ref. [45, 46]).

The production rate of gamma rays with energy $E_{\gamma}$ at the position $\vec{r}$ of the Galaxy, due to inverse Compton scattering of dark matter electrons (or positrons) with number density $f_{e^{ \pm}}\left(E_{e}, \vec{r}\right)$ on photons of the ISRF with number density $f_{\operatorname{ISRF}}(\epsilon, \vec{r})$, is given by

$$
\begin{aligned}
& \frac{d R_{\gamma}^{\mathrm{IC}}(\vec{r})}{d E_{\gamma}}= \\
& \int_{0}^{\infty} d \epsilon \int_{m_{e}}^{\infty} d E_{e} \frac{d \sigma^{\mathrm{IC}}\left(E_{e}, \epsilon\right)}{d E_{\gamma}} f_{e^{ \pm}}\left(E_{e}, \vec{r}\right) f_{\mathrm{ISRF}}(\epsilon, \vec{r}) .
\end{aligned}
$$

Here, $d \sigma^{\mathrm{IC}} / d E_{\gamma}$ denotes the differential cross section of inverse Compton scattering of an electron with energy $E_{e}$, where an ISRF photon with energy $\epsilon$ is up-scattered to energies between $E_{\gamma}$ and $E_{\gamma}+d E_{\gamma}$. It can be derived from the Klein-Nishina formula and is given by

$$
\begin{aligned}
& \frac{d \sigma^{\mathrm{IC}}\left(E_{e}, \epsilon\right)}{d E_{\gamma}}=\frac{3}{4} \frac{\sigma_{\mathrm{T}}}{\gamma_{e}^{2} \epsilon} \times \\
& \times\left[2 q \ln q+1+q-2 q^{2}+\frac{1}{2} \frac{(q \Gamma)^{2}}{1+q \Gamma}(1-q)\right],
\end{aligned}
$$

where $\sigma_{\mathrm{T}}=0.67$ barn denotes the Compton scattering cross section in the Thomson limit, $\gamma_{e} \equiv E_{e} / m_{e}$ is the Lorentz factor of the electron, $m_{e}=511 \mathrm{keV}$ is the electron mass, and we have defined $\Gamma \equiv 4 \gamma_{e} \epsilon / m_{e}$ and $q \equiv E_{\gamma} / \Gamma\left(E_{e}-E_{\gamma}\right)$. Eq. (6) holds in the limit where $\epsilon, m_{e} \ll E_{e}$, and kinematics and the neglect of downscattering require that $\epsilon \leq E_{\gamma} \leq\left(1 / E_{e}+1 / 4 \gamma_{e}^{2} \epsilon\right)^{-1} \equiv$ $E_{\gamma}^{\max }$. In the calculations we will further assume that the photon and electron fields are isotropic; taking into account the anisotropy of the photons, which are mainly produced in the Galactic disk, would give $\mathcal{O}(10 \%-20 \%)$ corrections to the ICS fluxes [47].

The gamma-ray flux from ICS that is received at Earth reads

$$
\frac{d J_{\text {halo-IC }}}{d E_{\gamma}}(l, b)=2 \cdot \frac{1}{4 \pi} \int_{0}^{\infty} d s \frac{d R_{\gamma}^{\mathrm{IC}}[r(s, l, b)]}{d E_{\gamma}},
$$

where the factor of 2 takes into account the fact that both dark matter electrons and positrons contribute equally to the total flux of gamma rays.

For the number density of ISRF photons we will use results from Ref. [48]. On the other hand, the number density of electrons and positrons from dark matter decay follows from solving the appropriate transport equation, which incorporates the effects of diffusion, reacceleration and convection in the Galactic magnetic field and energy losses due to synchrotron emission and inverse Compton scattering on the ISRF (see e.g. [49, 50, 51]). However, at higher energies above a few $10 \mathrm{GeV}$ the transport equation is dominated by the energy loss terms, and the number density of electrons and positrons can be approximated by

$$
f_{e^{ \pm}}\left(E_{e}, \vec{r}\right)=\frac{1}{b\left(E_{e}, \vec{r}\right)} \frac{\rho_{\text {halo }}(\vec{r})}{m_{\mathrm{dm}} \tau_{\mathrm{dm}}} \int_{E_{e}}^{\infty} d \tilde{E}_{e} \frac{d N_{e^{ \pm}}}{d \tilde{E}_{e}} .
$$

Here, $b\left(E_{\mathrm{e}}, \vec{r}\right)$ accounts for the energy losses and contains a part that comes from ICS on the ISRF, and a part that comes from synchrotron losses in the Galactic magnetic field, $b=b_{\mathrm{ICS}}+b_{\mathrm{syn}}$. We set $f_{e^{ \pm}}=0$ outside of the diffusion zone, which we model by a cylinder of half-height $L=3 \mathrm{kpc}$ and radius $R=20 \mathrm{kpc}^{1}{ }^{1}$ The impact of a variation of the height of the diffusive halo on our results will be discussed below. by

The part of the energy loss that is due to ICS is given

$$
\begin{aligned}
& b_{\mathrm{ICS}}\left(E_{e}, \vec{r}\right)= \\
& \int_{0}^{\infty} d \epsilon \int_{\epsilon}^{E_{\gamma}^{\max }} d E_{\gamma}\left(E_{\gamma}-\epsilon\right) \frac{d \sigma^{\mathrm{IC}}\left(E_{e}, \epsilon\right)}{d E_{\gamma}} f_{\mathrm{ISRF}}(\epsilon, \vec{r}) .
\end{aligned}
$$

For electron energies $E_{e}=1 \mathrm{GeV}, b_{\mathrm{ICS}}$ ranges between $4.1 \times 10^{-17} \mathrm{GeV} \mathrm{s}^{-1}$ and $1.9 \times 10^{-15} \mathrm{GeV} \mathrm{s}^{-1}$, depending on $\vec{r}$. At higher energies $b_{\text {ICS }}$ approximately scales like $\sim E_{e}^{2}$. On the other hand, the synchrotron loss part reads

$$
b_{\mathrm{syn}}\left(E_{e}, \vec{r}\right)=\frac{4}{3} \sigma_{\mathrm{T}} \gamma_{e}^{2} \frac{B^{2}}{2},
$$

where $B^{2} / 2$ is the energy density of the Galactic magnetic field, and we set $B=6 \mu \mathrm{G} \exp (-|z| / 5 \mathrm{kpc}-$ $r / 20 \mathrm{kpc})$ for definiteness [45]. At position of the Sun this yields an energy loss of $b_{\mathrm{syn}} \simeq 4.0 \times$ $10^{-17}\left(E_{e} / \mathrm{GeV}\right)^{2} \mathrm{GeV} \mathrm{s}^{-1}$.

Substituting Eq. (8) into Eq. (5), the flux from inverse Compton scattering of electrons and positrons from dark matter on the Galactic radiation field can be calculated.

In addition to the gamma-ray fluxes that originate from the decay of dark matter particles in the Milky Way halo, there exists a largely isotropic contribution generated by the decay of dark matter particles at cosmological distances. Analogously to the Milky Way component, the latter receives contributions from the direct decay of dark matter particles into photons, and from the gamma rays produced by the inverse Compton scattering of dark

\footnotetext{
1 For some sample decay channels we have cross-checked with GALPROP v50p, using appropriately modified versions of the model 50P_599278 (which adopts a diffusive halo with $L=4 \mathrm{kpc}$ ) and of the annihilation package, that our approximations give correct ICS gamma-ray sky maps at the $30 \%$ level everywhere in the sky for gamma-ray energies above $1 \mathrm{GeV}$.
} 
matter electrons and positrons on the intergalactic radiation field.

The direct decay of dark matter particles at cosmological distances produces a gamma-ray flux that is given by

$$
\begin{aligned}
& \frac{d J_{\mathrm{eg}}}{d E_{\gamma}}=\frac{\Omega_{\mathrm{dm}} \rho_{\mathrm{c}}}{4 \pi m_{\mathrm{dm}} \tau_{\mathrm{dm}}} \times \\
& \times \int_{0}^{\infty} d z \frac{1}{H(z)} \frac{d N_{\gamma}}{d E_{\gamma}}\left[(z+1) E_{\gamma}\right] e^{-\tau\left(E_{\gamma}, z\right)},
\end{aligned}
$$

where $H(z)=H_{0} \sqrt{\Omega_{\Lambda}+\Omega_{\mathrm{m}}(z+1)^{3}}$ is the Hubble expansion rate as a function of redshift $z$, and $\rho_{\mathrm{c}}=5.5 \times$ $10^{-6} \mathrm{GeV} / \mathrm{cm}^{3}$ denotes the critical density of the Universe. Throughout this work we assume a $\Lambda$ CDM cosmology with parameters $\Omega_{\Lambda}=0.74, \Omega_{\mathrm{m}}=0.26, \Omega_{\mathrm{dm}}=0.21$ and $h \equiv H_{0} / 100 \mathrm{~km} \mathrm{~s}^{-1} \mathrm{Mpc}^{-1}=0.72$, as derived from the five-year WMAP data [19].

The extragalactic ICS radiation of electrons and positrons from dark matter is expected to come mainly from scattering on the CMB [39]. Note that there is a similar component that comes from electrons and positrons produced in the Milky Way halo, but outside of the diffusion zone (see Ref. [40] for the analogous case of annihilation). For dark matter particles with masses below $3-5 \mathrm{TeV}$ these components are all expected to become relevant only for gamma-ray energies $E_{\gamma} \lesssim 10 \mathrm{GeV}$. We will neglected extragalactic ICS radiation from dark matter decay in the present work. Note, however, that this radiation would be largely isotropic and would somewhat reduce the overall anisotropy of the ICS radiation at these lower energies.

In Eq. (11) we included an attenuation factor for the gamma-ray flux, which incorporates the effects of electron-positron pair production by collisions of gamma rays from dark matter decay with the extragalactic background light emitted by galaxies in the ultraviolet, optical and infrared frequencies [52]. The attenuation factor is determined by the optical depth $\tau\left(E_{\gamma}, z\right)$, for which we will use the results from [53] throughout this work. ${ }^{2}$ In Fig. 1 we show isocontours of the optical depth in the redshift vs. energy plane. It is apparent from the plot that gamma rays with high energies around $1 \mathrm{TeV}$ are strongly attenuated and come mainly from redshifts $z \lesssim 0.05$. On the other hand, the flux of gamma rays originating from the decay of dark matter particles in the Galactic halo is barely attenuated by pair production on the ISRF at energies below $10 \mathrm{TeV}$ [55].

To compare the sizes of the halo and the extragalactic component of gamma rays from decaying dark matter (neglecting ICS radiation for simplicity), we show in

\footnotetext{
${ }^{2}$ In Ref. [53] the optical depth is calculated for redshifts $z<5$. Following Ref. [54], we assume that the optical depth does not increase beyond $z=5$ and set $\tau\left(E_{\gamma}\right)_{z>5}=\tau\left(E_{\gamma}\right)_{z=5}$.
}

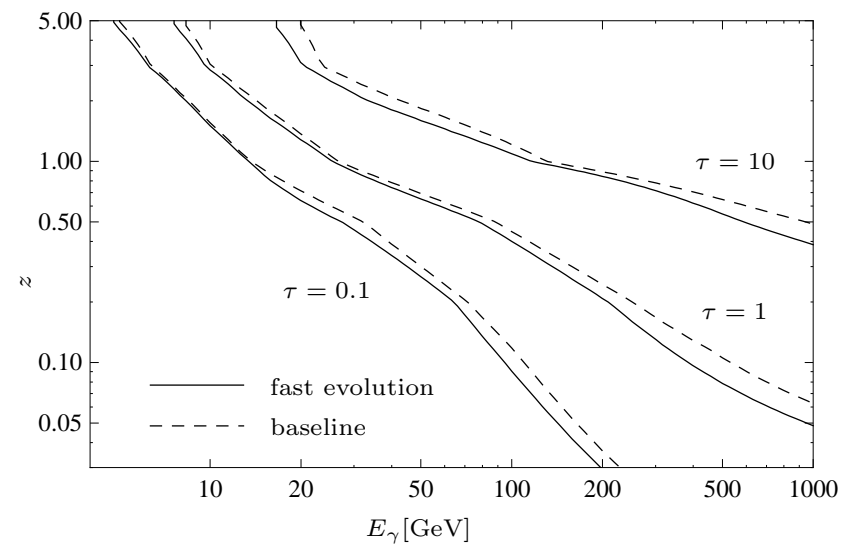

FIG. 1: Isocontours of the optical depth $\tau\left(E_{\gamma}, z\right)$ of gammaray photons, emitted at redshift $z$ and observed at Earth with energy $E_{\gamma}$. We show results for two different models of the intergalactic background light $[53$. Throughout this work, we will adopt the "fast evolution" model.

Fig. 2 the total flux of photons, integrated over all energies, as a function of the angular distance $\psi$ from the Galactic center, for a dark matter particle which decays producing a monoenergetic photon with an energy in the range $E_{\gamma}^{\prime} \simeq 10 \mathrm{GeV}-1 \mathrm{TeV}$. As apparent from the figure, the cosmological contributions decrease with energy due to the attenuation described above, while the radiation profile from decaying particles in the halo is independent of energy. The halo contribution typically dominates the total flux independently of the halo profile, except at low energies $E_{\gamma}^{\prime} \lesssim 10 \mathrm{GeV}$ in the direction of the Galactic anticenter. The differences in the two dark matter profiles become only relevant near the Galactic center when $\psi \lesssim 10^{\circ}$, and at the Galactic center the flux predicted for the Einasto profile is almost one order of magnitude larger than the corresponding flux from the isothermal profile.

\section{LARGE-SCALE ANISOTROPIES IN GAMMA RAYS FROM DECAYING DARK MATTER}

The decay of dark matter particles can produce gamma rays that could be detected as an exotic contribution to the diffuse extragalactic gamma-ray background (EGBG). The diffuse extragalactic background at high energies is believed to be dominated by the emission from unresolved active galactic nuclei and is expected to approximately follow a simple power law, with an intensity and index that has to be determined by fitting to the data [56, 57, 58]. Thus, if dark matter particles decay at a sufficiently fast rate, one generically expects to observe a deviation from a simple power law in the gamma-ray energy spectrum, which could show up in experiments like Fermi LAT. 


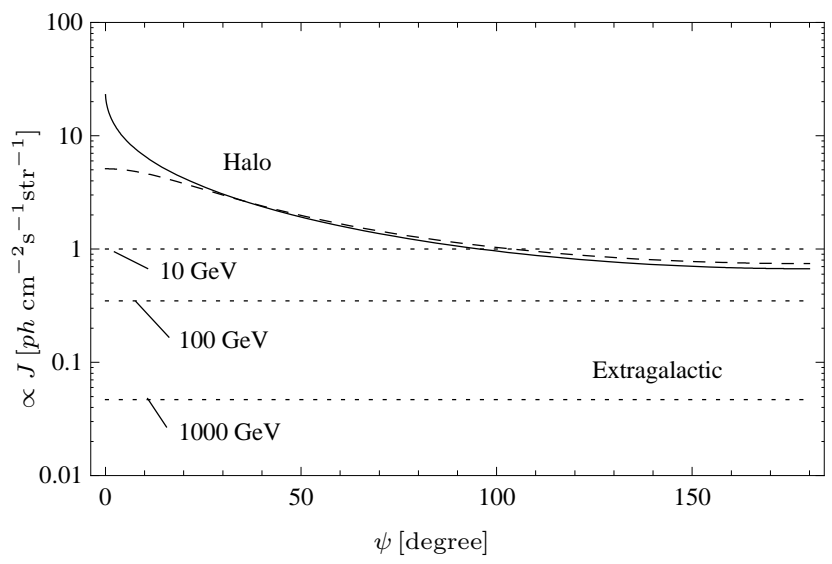

FIG. 2: Angular profile of the gamma-ray signal from dark matter decay as function of the angle $\psi$ to the center of the galaxy. The solid (dashed) line shows the contribution from decay in the Milky Way halo, assuming the Einasto (isothermal) profile. Extragalactic contributions are shown in dotted lines for the case that dark matter decay produces a monoenergetic line with energies between $E_{\gamma}^{\prime}=10$ and $1000 \mathrm{GeV}$. The fluxes are integrated over energy and normalized to the size of the extragalactic component when absorption is neglected.

A complementary signature of dark matter decay is the observation of anisotropies in the EGBG. It is well known that the offset between Sun and Galactic center causes a peculiar angular dependence in the gamma-ray signal from dark matter decaying [33] (or annihilating [59, 60]) in the Milky Way halo, even if the halo profile itself is isotropic. The halo signal is largest in direction of the Galactic center and smallest in direction of the Galactic anticenter (cf. Fig. 2). The observation of an anisotropy that is aligned in this way would be a strong signal for a contribution from dark matter, and, on the other hand, its non-observation would provide strong constraints. Gamma rays from the decay of dark matter particles at cosmological distances are isotropic and tend to reduce the anisotropy of the overall signal. This attenuation effect is however always small, due to the relative weakness of the extragalactic component.

To analyze the prospects of detecting a gamma-ray anisotropy from dark matter decay at the Fermi LAT it is convenient to define the quantity

$$
A_{b_{0}: b_{1}}=\frac{\bar{J}_{\mathrm{GC}}-\bar{J}_{\mathrm{GAC}}}{\bar{J}_{\mathrm{GC}}+\bar{J}_{\mathrm{GAC}}}
$$

where $\bar{J}_{\mathrm{GC}}$ and $\bar{J}_{\mathrm{GAC}}$ in general denote the total diffuse gamma-ray flux (from dark matter and from astrophysical sources) integrated over $E_{\gamma}$ in some energy range, and averaged over the hemisphere in direction of the Galactic center (GC) and anticenter (GAC), respectively. Sky regions with small, $|b|<b_{0}$, or large, $|b|>b_{1}$, Galactic latitudes are excluded from the average.

\begin{tabular}{c|c|c}
\hline \hline $\begin{array}{c}\text { Sky patch } \\
b_{0}: b_{1}\end{array}$ & \multicolumn{2}{|c}{ Anisotropy $A_{b_{0}: b_{1}}$} \\
\hline \hline $10^{\circ}: 90^{\circ}$ & $0.21-0.36$ & $0.20-0.33$ \\
$10^{\circ}: 20^{\circ}$ & $0.32-0.50$ & $0.29-0.45$ \\
$20^{\circ}: 60^{\circ}$ & $0.21-0.35$ & $0.20-0.33$ \\
$60^{\circ}: 90^{\circ}$ & $0.07-0.13$ & $0.07-0.13$ \\
\hline \hline
\end{tabular}

TABLE I: Anisotropy of the gamma-ray signal from the decay $\psi \rightarrow \gamma \nu$ of a fermionic dark matter particle $\psi$, after subtracting astrophysical contributions. The ranges correspond to the anisotropies from gamma-ray lines with energies between $10 \mathrm{GeV}$ and $1000 \mathrm{GeV}$ in different regions of the sky, see Eq. (12).

As an example, we calculate the anisotropy parameter $A$ in different regions of the sky for the decay $\psi \rightarrow \gamma \nu$ of a fermionic dark matter particle $\psi$, after subtracting astrophysical sources (the Galactic foreground and the extragalactic gamma-ray background), in order to compare the anisotropy expected purely from dark matter decay to the anisotropy expected from the Galactic models (in the rest of the paper, however, we will consider both sources of gamma rays simultaneously, calculating the anisotropy of the total flux). In this case, the energy spectrum of gamma rays has two components: a monoenergetic line from the decay of dark matter particles in the halo and a redshifted line from decays at cosmological distances (note that in this decay channel there is no contribution from ICS). We show in Tab. [ the values of $A$ for dark matter particles with masses between $20 \mathrm{GeV}$ and $2 \mathrm{TeV}$, producing monoenergetic photons with energies $10 \mathrm{GeV}$ and $1 \mathrm{TeV}$, respectively. It is interesting that the anisotropy parameter can be as large as 0.5 for large energies and relatively low latitudes. In the region defined by $b_{0}=10^{\circ}$ and $b_{1}=90^{\circ}$ (on which we will concentrate below), the anisotropy of the "pure" dark matter signal ranges between 0.20 and 0.36 , with only little dependence on the profile of the dark matter halo. These values have to be compared with the anisotropies of the Galactic foreground as predicted by GALPROP (see below), which are considerably smaller, and typically $A \lesssim 0.10$ in all the regions that are shown in Tab. I up to energies above $300 \mathrm{GeV}$. Furthermore, the anisotropies measured by EGRET for energies below $10 \mathrm{GeV}$ are consistent with the predictions for the Galactic foreground [61].

From the theoretical point of view, the search for anisotropies in the gamma-ray flux is a cleaner method for the indirect detection of dark matter than the search for an excess in the spectrum of the EGBG. As mentioned above, the genuinely extragalactic flux from active galactic nuclei and other extragalactic sources is very poorly understood. Thus, it is difficult to make firm predictions for the total gamma-ray flux in scenarios with decaying dark matter, even when the particle 
physics model is specified (namely, the dark matter mass, lifetime and decay modes). Moreover, there are other potentially important isotropic contributions to the total flux with an intensity that cannot be predicted theoretically. For instance, interactions of high energy cosmic rays with debris in the hypothetical Oort cloud could produce a sizable gamma-ray flux, provided that the column density is larger than $10^{-3} \mathrm{~g} \mathrm{~cm}^{-2}$ [62]. Since all these contributions to the total flux are perfectly isotropic, they cancel out when calculating the difference of the fluxes between the Galactic center and the Galactic anticenter regions.

To illustrate the large-scale anisotropy $A$ that could be produced by dark matter decay, we will show predictions for different dark matter decay channels and masses, including Galactic foreground radiation and the astrophysical extragalactic background. As Galactic foreground we take predictions of the conventional GALPROP-model as presented in Ref. 63] (model 44_500180), and as astrophysical EGBG we will use the following parameterization, which agrees with preliminary results from Fermi LAT [64]

$\frac{d J_{\mathrm{EGBG}}}{d E_{\gamma}}=5.8 \times 10^{-7}\left(\mathrm{GeV} \mathrm{cm}^{2} \mathrm{sstr}\right)^{-1}\left(\frac{E_{\gamma}}{1 \mathrm{GeV}}\right)^{-2.45}$.

We show in the left panels of Fig. 3 the predicted anisotropy of the total gamma-ray flux that would be measurable in different regions of the sky if the dark matter particles decay exclusively into $\tau^{+} \tau^{-}$pairs. The anisotropy is calculated with taking into account the galactic foreground and the extragalactic gamma-ray background as discussed above. The dark matter mass is taken to be $m_{\mathrm{dm}}=600 \mathrm{GeV}$, and we set the lifetime to $\tau_{\mathrm{dm}} \simeq 3.5 \times 10^{27} \mathrm{~s}$. The energy spectra $d N_{\gamma, \mathrm{e}} / d E_{\gamma, \mathrm{e}}$ of the photons, electrons and positrons produced in the decay are calculated with the event generator PYтнIA 6.4 [65]. The lifetime is chosen such that the gamma-ray fluxes are below and compatible with the EGBG, as demonstrated in the right panels of Fig. 31, where we also show preliminary results from the Fermi LAT for comparison. ${ }^{3}$ Furthermore, we note that the contribution to the local electron and positron fluxes from dark matter decay is negligible in this scenario. Interestingly, for the adopted choice of parameters, an anisotropy is predicted that is significantly different to the one expected from the diffuse Galactic emission in the conventional Galprop model. Such an anisotropy should, moreover, be observable by Fermi LAT, as illustrated by the boxes in the figure, which correspond to our estimates of the one-year and

\footnotetext{
${ }^{3}$ Fitting the preliminary Fermi LAT results with a Galactic foreground model is well beyond the scope of this paper. Hence, there is a mismatch between the total fluxes and the data, which does not affect our conclusions.
}

five-year statistical errors of Fermi LAT, assuming exposures of $\varepsilon=3 \times 10^{10} \mathrm{~cm}^{2} \mathrm{~s}[64]$ and $\varepsilon=2 \times 10^{11} \mathrm{~cm}^{2} \mathrm{~s}$ [67], respectively (for a discussion about our calculation of the statistical errors see below and appx. A). As expected, the size of the anisotropy is largest at low latitudes $b \lesssim 20^{\circ}$, and decreases slowly when considering higher latitudes. On the other hand, the statistical error is smallest in the sky-patch shown in the upper panels of Fig. 3, where the whole sky with $|b| \geq 10^{\circ}$ is included. The effects of ICS radiation are negligible in the present case of decay into $\tau^{+} \tau^{-}$pairs, since the electrons and positrons produced in the subsequent decay of the taus have only relatively small energies.

To illustrate the impact of ICS radiation on the anisotropy parameter $A$, we show in Fig. 4 the anisotropy of the gamma-ray flux assuming that the dark matter particle decays into $e^{+} e^{-}$pairs (with $m_{\mathrm{dm}}=1000 \mathrm{GeV}$ and $\tau_{\mathrm{dm}}=2 \times 10^{27} \mathrm{~s}$ ). In this case the dominant source of gamma rays is inverse Compton scattering (note that we assumed that the decaying dark matter particle has spin 1; for scalar dark matter particles, helicity suppression leads to an enhanced production of final-state radiation [66], weakening the relative contribution from ICS). For reference, we also show the anisotropy that would be measurable if ICS radiation were absent (dashed lines in left panels of Fig. (4). Note that in this scenario electrons and positrons produced in the dark matter decay give a sizeable contribution to the local cosmic-ray fluxes, without being in conflict with the PAMELA and Fermi LAT data. Again, we find that a sizable anisotropy is expected in several patches of the sky. In this case, however, the gamma rays relevant for our predictions are mainly produced close to the Galactic center, above and below the Galactic disk. Hence, the anisotropies are relatively weak at higher latitudes $|b| \gtrsim 20^{\circ}$. A more detailed discussion about the differences between prompt and ICS gamma rays from dark matter decay can be found in appx. $\mathrm{B}$

If dark matter decay is the origin of the excess in the positron fraction observed by PAMELA and in the total electron-plus-positron flux observed by the Fermi LAT, the predicted anisotropies in the gamma-ray flux can be quite large. In Fig. 5 we show our results for the anisotropy, $A$, which is expected to be observed by the Fermi LAT if the dark matter particle decays via one of the five different decay channels that were found in Ref. [15] to fit well the positron and electron data. ${ }^{4}$ To minimize the statistical errors, we concentrate on the region defined by $b_{0}=10^{\circ}$ and $b_{1}=90^{\circ}$ (see appx. (B). As apparent from the plots, the predictions for some decay channels (namely the decay into $W^{ \pm} \mu^{\mp}$ ) are al-

\footnotetext{
${ }^{4}$ We use slightly larger lifetimes than the ones given in Ref. 15 to account for the larger value for the local dark matter density used in this work.
} 

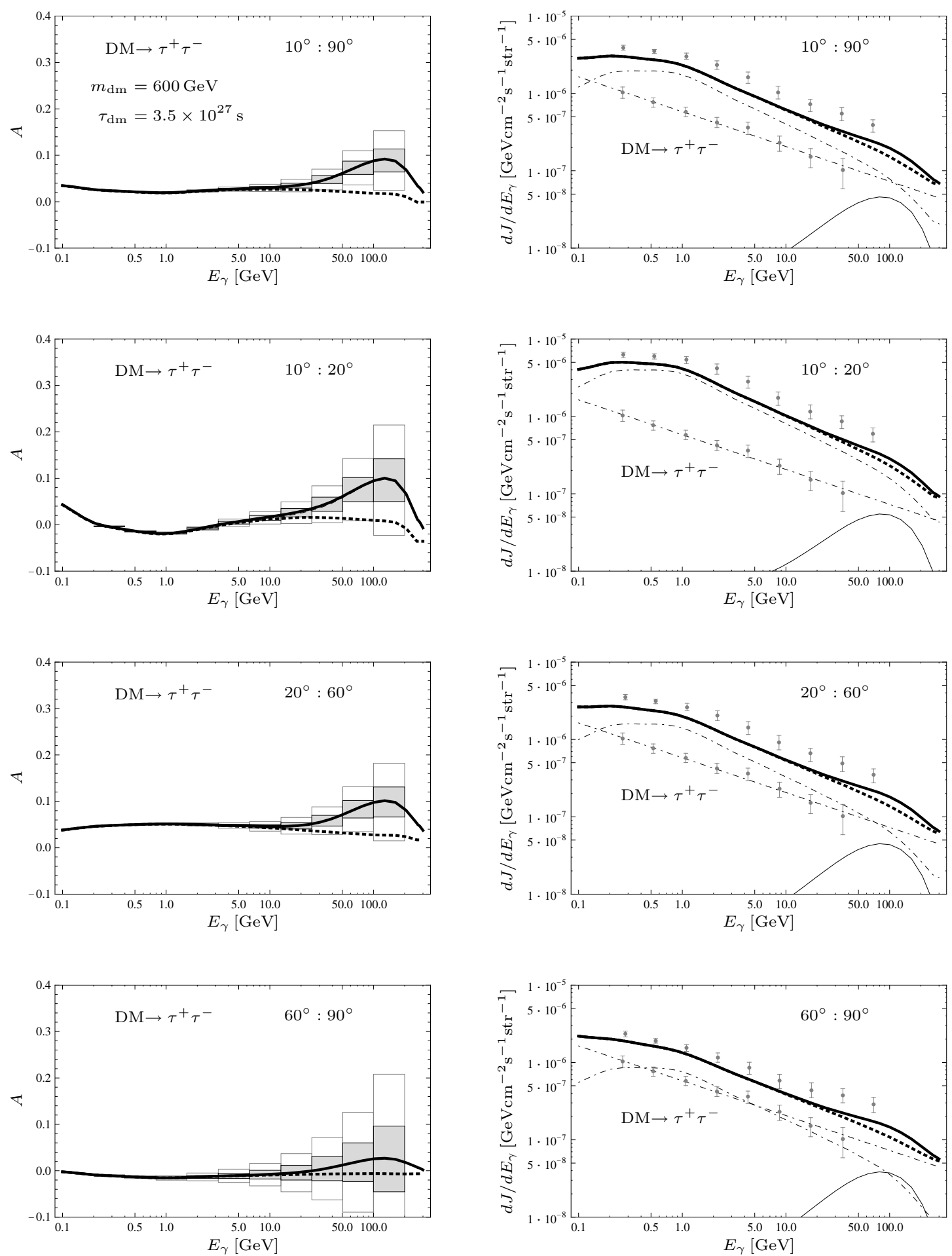

FIG. 3: Left panels: Anisotropy of the gamma-ray flux from dark matter decay into $\tau^{+} \tau^{-}$pairs as a function of energy. The dotted line shows the background anisotropy as expected from the Galactic foreground, while the solid line shows the anisotropy of signal + background. We also show the signal + background anisotropy neglecting gamma rays from ICS (dashed, overlapping with solid line). The boxes show estimates of the statistical errors for one-year and five-year Fermi LAT observations. Right panels: Gamma-ray fluxes averaged over all Galactic longitudes as a function of energy. The thin solid line shows the gamma rays from dark matter decay. The two dash-dotted lines show the astrophysical EGBG and the Galactic foreground separately. The thick solid line shows the sum of all contributions, whereas the dotted line shows the sum without contributions from dark matter. From top to bottom the different panels show predictions for different patches of the sky. The data points show preliminary Fermi LAT data [64] for the total diffuse flux (upper points, in the $10^{\circ}: 90^{\circ}$ region we averaged the presented preliminary results appropriately) and the EGBG (lower points). 
ready in conflict with the preliminary results of the Fermi LAT collaboration for the EGBG, whereas other decay channels are marginally consistent (e.g. the decay into $\mu^{+} \mu^{-}$pairs). However, even for those channels which are compatible with the data, sizeable anisotropies, around $A \simeq 0.2-0.3$, are predicted at energies $E_{\gamma} \simeq 100 \mathrm{GeV}$. This is significantly different from the anisotropy expected for the astrophysical foreground. As indicated by our estimates of the statistical error bars for oneyear Fermi LAT data taking, this deviation should be clearly visible in the upcoming results for the diffuse gamma-ray sky. On the other hand, its non-observation would set very strong constraints on the decaying dark matter interpretation of the positron excess observed by PAMELA/Fermi LAT.

Uncertainties in the determination of the above large-scale anisotropy come from different sources. When neglecting ICS radiation, the prediction of an anisotropy between 0.2 and 0.3 in the dark matter signal at latitudes $|b|>10^{\circ}$ is relatively robust. The main sources of uncertainty are the profile of the Milky Way dark matter halo and its normalization. As discussed above, the dependence on the profile is rather weak (cf. Tab. I). Only in case of a much lower value for the local dark matter density, say $\rho_{\odot}=0.2 \mathrm{GeV} \mathrm{cm}^{-3}$, and only for gamma rays with energies $E_{\gamma}^{\prime} \lesssim 10 \mathrm{GeV}$, the anisotropy can become as small as $A \simeq 0.15$. On the other hand, the size and anisotropy of the ICS radiation from electrons and positrons originating from dark matter decay is plagued by many uncertainties like the exact height of the diffusion zone, the distribution of the ISRF and the size of the Galactic magnetic field. In general the ICS radiation, and hence the overall anisotropy of the observed flux, becomes stronger if the height of the diffusive halo is increased, but a detailed study of these uncertainties is beyond the scope of this paper. Note, however, that the large scale anisotropies predicted for the decay channels shown in Fig. 5 are sizeable even if ICS radiation is neglected (dashed lines in left panels).

Finally we will discuss in a more quantitative way the main prospects for the Fermi LAT to detect gamma rays from dark matter decay through the observations of large-scale anisotropies. To this end we will neglect inverse Compton radiation from the electrons and positrons produced in the decay of the dark matter particle, and we will assume perfect subtraction of the Galactic foreground. The remaining flux is then expected to be constituted by the isotropic EGBG, which is possibly contaminated by anisotropic radiation from dark matter decay. For definiteness, we will assume that the remaining flux follows the power law in Eq. (13).

Provided that a fraction $f_{\mathrm{s}}$ of the considered gammaray photons in a given energy range is due to decaying dark matter, the measured anisotropy $A$ is given by

$$
A=f_{\mathrm{s}} A_{\mathrm{s}}+\left(1-f_{\mathrm{s}}\right) A_{\mathrm{bg}} .
$$

Here, $A_{\mathrm{s}}$ and $A_{\mathrm{bg}}$ denote the anisotropy of the dark matter signal, which can be read off from Tab. \. and the anisotropy of the astrophysical background, which is $A_{\mathrm{bg}}=0$ in our case, respectively. A possible detection at the $3 \sigma$-level requires that

$$
f_{\mathrm{s}}>\frac{3 \sigma_{A}}{A_{\mathrm{s}}-A_{\mathrm{bg}}},
$$

where $\sigma_{A}$ denotes the standard deviation of the anisotropy $A$. It depends on the total number of measured photons, $N_{\gamma}$ and can be approximated by $\sigma_{A} \simeq$ $N_{\gamma}^{-1 / 2}$. The adopted approximation for $\sigma_{A}$ is better than $10 \%$ as long as $|A| \lesssim 0.3$ and $\sigma_{A} \lesssim 0.2$. See appx. A for a short discussion. The photon number is given by

$$
N_{\gamma}=\varepsilon \cdot \Omega_{\mathrm{sky}} \int_{E_{0}}^{E_{1}} d E_{\gamma} \frac{d J}{d E_{\gamma}},
$$

where $\varepsilon$ denotes the experimentally given exposure (see above) and $\Omega_{\text {sky }}$ is the solid angle of the observed sky, which is given by $\Omega_{\text {sky }}=0.83 \cdot 4 \pi$ if the Galactic disk with $|b|<10^{\circ}$ is excluded. Following Eq. (13) the Fermi LAT will detect $N_{\gamma} \simeq 3.0 \times 10^{4}\left(N_{\gamma} \simeq 1.1 \times 10^{3}\right)$ photons with energies $E_{\gamma} \geq 10 \mathrm{GeV}\left(E_{\gamma} \geq 100 \mathrm{GeV}\right)$ after five years of data taking. Taking for definiteness $A_{\mathrm{s}}=0.3$, this allows in principle a $3 \sigma$-detection of a dark matter contamination down to $f_{\mathrm{s}} \simeq 6 \%\left(f_{\mathrm{s}} \simeq 30 \%\right)$. Note, however, that additional statistical noise and systematic uncertainties from point source subtraction and the determination of the Galactic foreground are neglected and can reduce the sensitivity to $f_{\mathrm{s}}$ by factors of order one.

\section{ANGULAR POWER SPECTRUM OF GAMMA RAYS FROM DECAYING DARK MATTER}

We will now discuss the angular power spectrum $C_{\ell}$ of gamma rays which stem from the decay of dark matter particles (for the annihilation case, see e.g. 68, 69, 70, 71, 72, 73]). On large angular scales the angular power spectrum is expected to be completely dominated by the dipole-like asymmetry of the halo flux, which is - as discussed above - due to the offset between the Sun and the Galactic center. However, on smaller scales spatial fluctuations of the dark matter density can become relevant. These fluctuations are related to subhalos of our own Galactic halo, and to the large-scale structure of the dark matter distribution in the nearby Universe. The abundance and distribution of subhalos and their impact on indirect searches for dark matter is still a debated question [22, 74]. Recent $N$-body simulations suggest that inside a radius of $r=100 \mathrm{kpc}$ around the Galactic center, the fraction of the halo mass that is bound in subhalos can be as small as a few percent [35]. Such a small value would lead to 

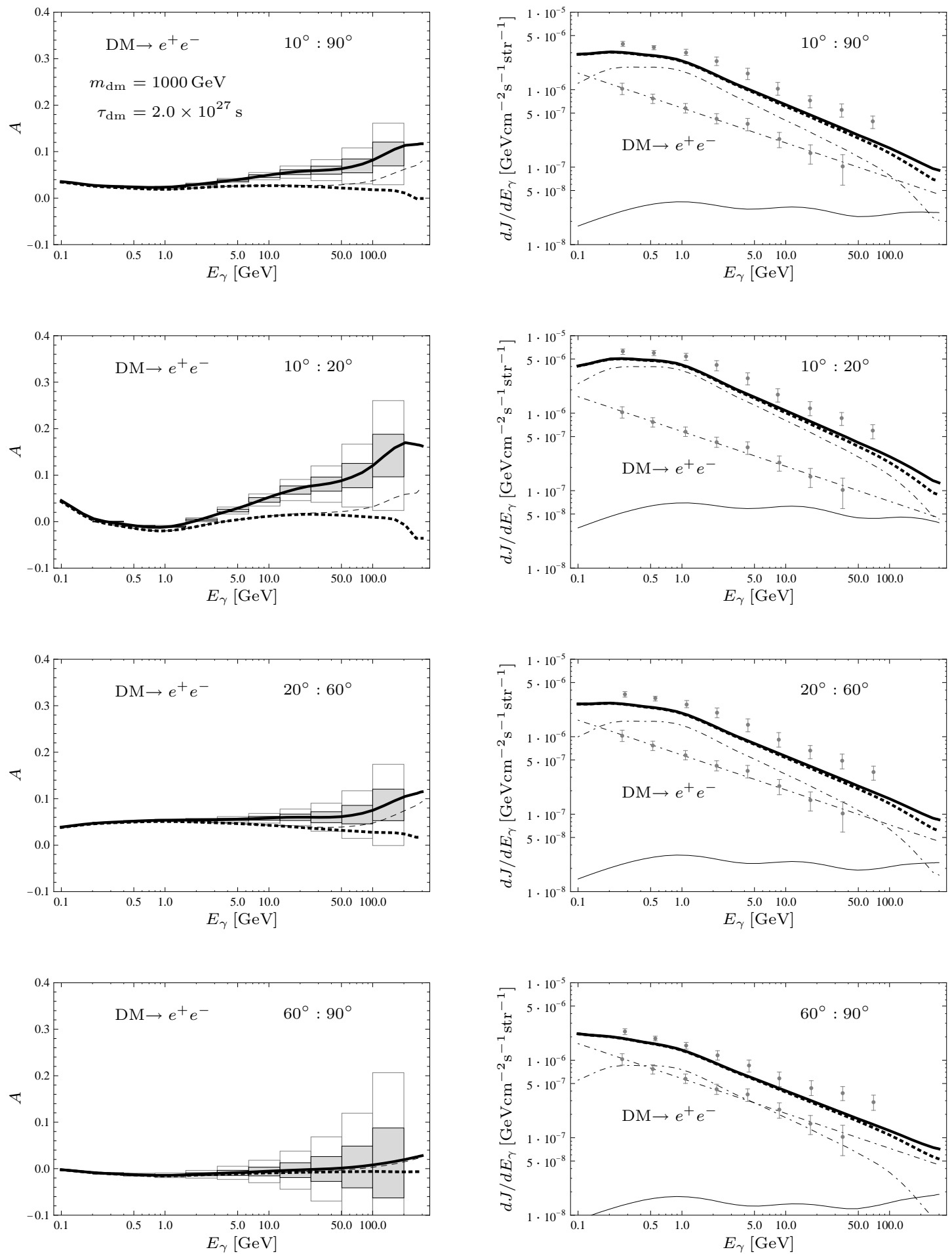

FIG. 4: Same as Fig. 3, but for decay into $e^{+} e^{-}$pairs. Here, in the left panels, the difference in the predictions for the anisotropy when including ICS radiation (solid line) and neglecting ICS radiation (dashed line) is clearly visible. 
a strong suppression of subhalo-related signals in case of decaying dark matter. In this work we will concentrate on the more robust predictions related to the large-scale structures of the dark matter distribution, and we will assume a smooth Milky Way halo when calculating the angular power spectrum. Furthermore, we neglect ICS radiation throughout this section.

The fluctuations of the dark matter gamma-ray flux in a certain energy band can be expanded in spherical harmonics $Y_{\ell m}$ with coefficients $a_{\ell m}$ according to

$$
\delta J(\vec{n}) \equiv J(\vec{n})-\langle J\rangle=\langle J\rangle \sum_{\ell m} a_{\ell m} Y_{\ell m}(\vec{n}) .
$$

Here $\langle J\rangle$ denotes the mean gamma-ray flux, averaged over the whole sky, and the coefficients $a_{\ell m}$ can be calculated from

$$
a_{\ell m}=\langle J\rangle^{-1} \int d \Omega_{\vec{n}} \delta J(\vec{n}) Y_{\ell m}^{*}(\vec{n}) .
$$

Our goal is to calculate the angular power spectrum $C_{\ell} \equiv\left\langle\left|a_{\ell m}\right|^{2}\right\rangle$, which can be split up into an extragalactic and a halo part according to

$$
C_{\ell}=f_{\mathrm{eg}}^{2} C_{\ell}^{\mathrm{eg}}+\left(1-f_{\mathrm{eg}}\right)^{2} C_{\ell}^{\text {halo }} .
$$

Here, $f_{\text {eg }}$ denotes the fraction of dark matter photons with extragalactic origin. Cross-correlations between the halo and the extragalactic component vanish in our case.

Following Eq. (18), the angular power spectrum of the halo component, $C_{\ell}^{\text {halo }}$, can be calculated by

$$
C_{\ell}^{\text {halo }} \propto\left|\int_{0}^{\pi} d \psi \sin \psi P_{\ell}^{0}(\cos \psi) J_{\text {halo }}(\psi)\right|^{2},
$$

where the $P_{\ell}^{m}$ denote the associated Legendre functions, $J_{\text {halo }}(\psi)$ is the angular profile of the halo flux as plotted in Fig. 2, and the power spectrum has to be normalized such that $C_{0}^{\text {halo }}=4 \pi$.

For the calculation of the extragalactic component of the angular power spectrum, $C_{\ell}^{\mathrm{eg}}$, it is convenient to define the window function

$$
W(z)=\frac{\Omega_{\mathrm{dm}} \rho_{\mathrm{c}}}{4 \pi m_{\mathrm{dm}} \tau_{\mathrm{dm}}} \int_{E_{0}}^{E_{1}} d E_{\gamma} \frac{d N_{\gamma}}{d E_{\gamma}}\left[(z+1) E_{\gamma}\right] e^{-\tau\left(E_{\gamma}, z\right)},
$$

for which holds that $\left\langle J_{\text {eg }}\right\rangle=\int_{0}^{\infty} d z W(z) / H(z)$ (cf. Eq. (11) above). The window function mainly depends on the energy spectrum of gamma rays from the dark matter decay $d N_{\gamma} / d E_{\gamma}$, and on the optical depth $\tau\left(E_{\gamma}, z\right)$. Following the Limber approximation, the angular power spectrum of the extragalactic component can be determined by (for details see e.g. Ref. [69])

$$
C_{\ell}^{\mathrm{eg}}=\left\langle J_{\mathrm{eg}}\right\rangle^{-2} \int_{0}^{\infty} \frac{d r}{r^{2}} W(z)^{2} P\left(k=\frac{\ell}{r}, z\right) .
$$

Here, $P(k, z)$ denotes the power spectrum of the dark matter distribution as a function of redshift $z$, and the comoving coordinate $r$ is related to $z$ by $d r=d z / H(z)$. The dark matter power spectrum at $z=0$ can be derived from $N$-body simulations or analytically in the Halo model, and we will adopt results from Ref. [54]. The corresponding redshift-dependence follows from the relation

$$
P(k, z)=P(k)_{z=0} \cdot D(z)^{2},
$$

where $D(z) \propto H(z) \int_{z}^{\infty} d z^{\prime}\left(1+z^{\prime}\right) H\left(z^{\prime}\right)^{-3}$ is the lineargrowth factor, normalized such that $D(0)=1$ 75]. In principle the above set of equations allow a calculation of the angular power spectrum of gamma rays for arbitrary gamma-ray spectra.

In Fig. 6 we show the quantity $W(z) / H(z)$ as a function of redshift, assuming that dark matter decay produces a monoenergetic line with energies between $E_{\gamma}^{\prime}=10 \mathrm{GeV}$ and $1 \mathrm{TeV}$. When normalized appropriately, $W(z) / H(z) \cdot d z$ gives the fraction of the received extragalactic dark matter photons that were emitted at redshifts $z \ldots z+d z$. As evident from the figure, the region of the Universe that contributes to the observable gamma-ray fluxes becomes larger for lower gamma-ray energies. At $E_{\gamma}^{\prime}=1 \mathrm{TeV}$, around $90 \%$ of the observable photons come from comoving distances $r \lesssim 400 \mathrm{Mpc}$, whereas at lower gamma-ray energies around $10 \mathrm{GeV}$ photons are received that come from distances up to $8 \mathrm{Gpc}$. Hence, in the latter case fluctuations in the extragalactic dark matter signal due to large-scale structures, which typically have sizes up to several $100 \mathrm{Mpc}$, are efficiently averaged out, which results in a correspondingly small value of $C_{\ell}^{\mathrm{eg}}$. For higher gammaray energies the probed region shrinks, yielding a larger $C_{\ell}^{\mathrm{eg}}$. Note, however, that the fraction of gamma rays from the decay of dark matter particles at cosmological distances, $f_{\text {eg }}$, presents an opposite energy dependence and decreases by an order of magnitude, from 0.42 to 0.03 , when increasing the gamma-ray energy from $E_{\gamma}^{\prime}=10 \mathrm{GeV}$ to $1 \mathrm{TeV}$. This behavior counteracts the growth of $C_{\ell}^{\mathrm{eg}}$ in Eq. (19).

Our results for the total angular power spectrum of gamma rays from decaying dark matter are shown in Fig. 7, including the halo and the extragalactic component, and assuming that the decay of the dark matter particle only produces monoenergetic photons with energies between $E_{\gamma}^{\prime}=10 \mathrm{GeV}$ and $1 \mathrm{TeV}$. As apparent from the plot, the halo component of $C_{\ell}$ increases slightly with $E_{\gamma}^{\prime}$, which is related to a corresponding decrease of $f_{\text {eg }}$ in Eq. (19). The variation of the extragalactic component with gamma-ray energy $E_{\gamma}^{\prime}$ is also on the $\mathcal{O}(1)$ level in most of the energy range, except at the highest energies $E_{\gamma}^{\prime} \gtrsim 1 \mathrm{TeV}$. In summary, the angular power spectrum looks qualitatively similar in the whole considered energy regime. The halo component dominates at large angles as long as $\ell \lesssim 100$, whereas the extragalactic component takes over 

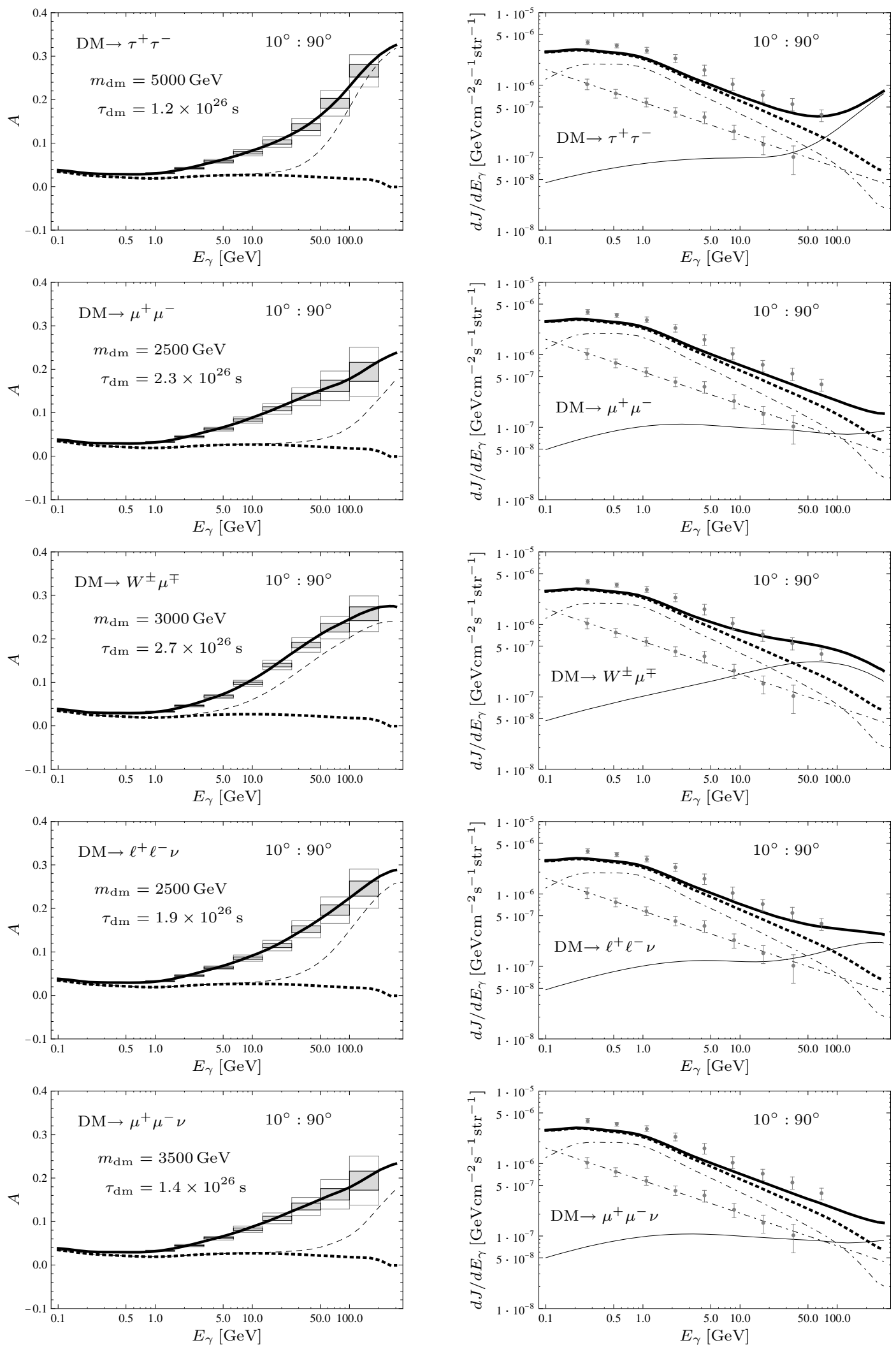

FIG. 5: Predictions for the five dark matter decay channels that where found to fit the positron excess as observed by PAMELA/Fermi. The left panels show predictions for the total measurable anisotropy at latitudes $|b|>10^{\circ}$ (solid line) and estimates of the corresponding 1-year and 5-year statistical error bars of Fermi LAT. The dotted line is the anisotropy predicted by GALPROP. In the right panels we show predictions for the averaged fluxes in the same sky region. Line coding is the same as in Fig. 3 


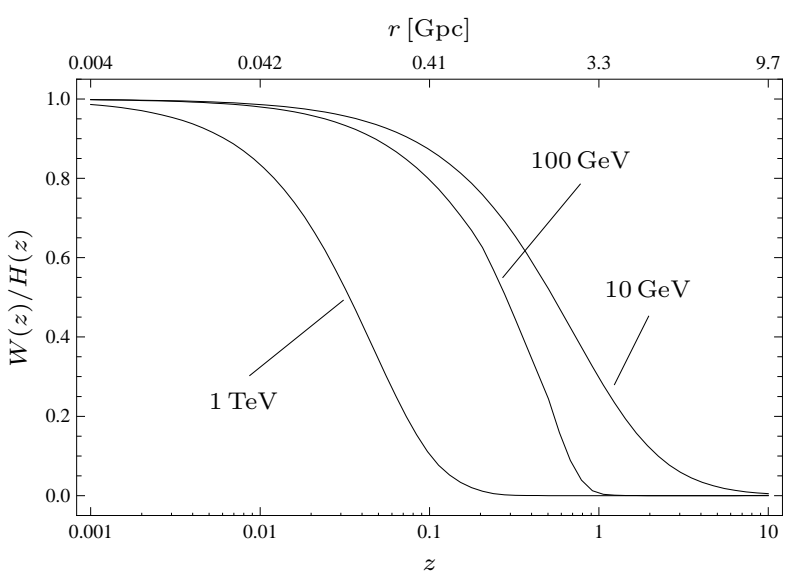

FIG. 6: Window function divided by Hubble rate, $W(z) / H(z)$, as a function of redshift $z$, with arbitrary normalization. We assume that dark matter decay is producing a monoenergetic line with energies between $E_{\gamma}^{\prime}=10 \mathrm{GeV}$ and $1 \mathrm{TeV}$. For comparison, we also show values for the comoving coordinate $r(z)$.

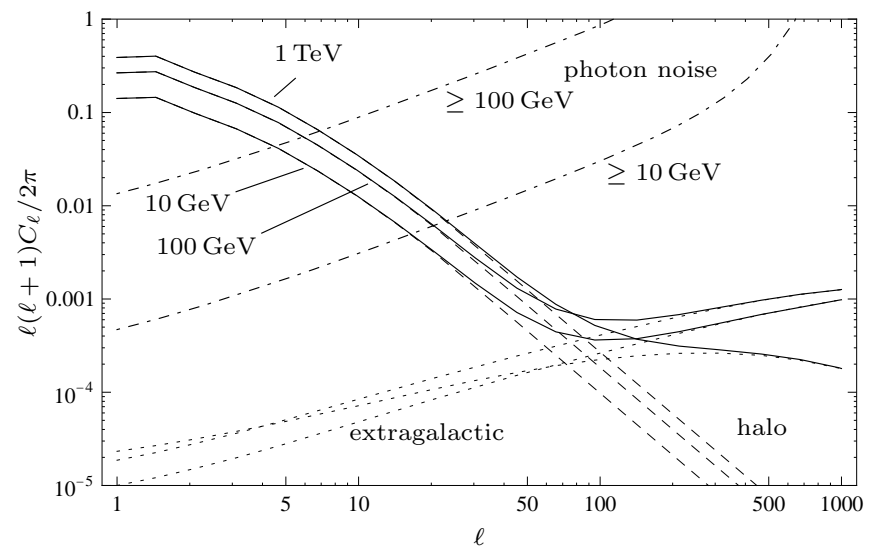

FIG. 7: Angular power spectrum of gamma-ray signal from decaying dark matter. Shown are the contributions from the halo component (dashed line) and the contribution from the extragalactic radiation (dotted line, based on results from [54]). The dash-dotted lines show for different gamma-ray energies our predictions of the photon noise level after five-years Fermi LAT observation.

at smaller angles with $\ell \gtrsim 100$, yielding values for the power spectrum around $\ell(\ell+1) C_{\ell} / 2 \pi \simeq \mathcal{O}\left(10^{-4}-10^{-3}\right)$.

To shortly discuss the observational prospects for Fermi LAT, we will simply assume that the total EGBG (as given by Eq. (13)) above a certain energy is due to dark matter decay. Then, following Ref. [69], the $1 \sigma$ deviation of the power spectrum, averaged over $\ell$-bins with size $\Delta \ell$, is given by

$$
\delta C_{\ell}=\sqrt{\frac{2}{(2 \ell+1) \Delta \ell f_{\mathrm{sky}}}}\left(C_{\ell}+\frac{C_{N}}{W_{\ell}^{2}}\right)
$$

where $C_{N}=4 \pi f_{\text {sky }} N_{\gamma}^{\text {tot }} /\left(N_{\gamma}^{\mathrm{s}}\right)^{2}$ is the photon noise, $N_{\gamma}^{\mathrm{s}}$ and $N_{\gamma}^{\text {tot }}$ denote the number of measured dark matter photons and number of total photons, respectively, $f_{\text {sky }}$ is the observed fraction of the sky and $W_{\ell}=\exp \left(-\sigma_{b}^{2} \ell^{2} / 2\right)$ is the window function of a Gaussian point-spread function. For the Fermi LAT we take as beam size $\sigma_{b}=0.1^{\circ},{ }^{5}$ and we set $\Delta \ell=0.5 \ell$. We only consider latitudes with $|b| \geq 20^{\circ}$, which implies that $f_{\text {sky }}=0.66$. In this part of the sky the ratio between the total gamma-ray fluxes and the EGBG flux is $\mathcal{O}(3)$ (see Fig. 5), and we will set $N_{\gamma}^{\text {tot }} / N_{\gamma}^{\mathrm{s}}=3$ for definiteness. The number of signal photons follows from Eqs. (11) and (16).

The resulting photon noise (second term of $\delta C_{\ell}$ in Eq. (24)) for five-years Fermi LAT observation is shown in Fig. 7 by the two dash-dotted lines, taking into account only gamma rays with energies $E_{\gamma} \geq 10 \mathrm{GeV}$ or $\geq 100 \mathrm{GeV}$, respectively. As apparent from these lines, the small-scale fluctuations are completely hidden under photon noise in the energy range we consider, and hence they are not accessible by current instruments like Fermi LAT. However, note that this is in contrast to gammaray signals from annihilating dark matter 71,73 . or different extragalactic astrophysical sources [6, 77], where the small-scale anisotropies can be orders of magnitude larger. This fact might eventually provide a handle on distinguishing the different scenarios, but a detailed investigation of the prospects is beyond the scope of this paper.

\section{CONCLUSIONS}

Dark matter particles could decay into gamma rays at a rate which is sufficiently large as to allow their indirect detection through an excess over the expected power law in the energy spectrum of the diffuse extragalactic gamma-ray background. In this work we have discussed a complementary way of indirectly detecting unstable dark matter particles by exploiting the fact that the Earth is not located at the center of the dark matter halo. We have discussed the relative size of the extragalactic and the halo component of the gamma rays from dark matter decay, incorporating the attenuation effects from pairproduction on the intergalactic background light, and we have calculated the dipole-like anisotropy between the high latitude gamma-ray flux coming from Galactic center and from Galactic anticenter regions for different decay channels. We have furthermore demonstrated the strong impact of gamma-rays from inverse Compton scattering of electrons and positrons from dark matter decay on the anisotropy signal. We have found that if dark matter decay is the correct explanation of the excesses in the positron fraction and the total electron-plus-positron flux reported by the PAMELA and Fermi LAT collabo-

\footnotetext{
${ }^{5}$ See http://www-glast.slac.stanford.edu
} 
rations, such an anisotropy in the gamma-ray flux should be observed by the Fermi LAT (see Fig. [5).

Lastly, we have calculated the angular power spectrum of the gamma-ray signal from dark matter decay, which exhibits imprints from the large scale structure of the Universe (see Fig. 7). We have shown, however, that for gamma ray energies above a few $\mathrm{GeV}$ these small scale effects are too weak to be observed with present instruments.

\section{Acknowledgments}

C.W. would like to thank Luca Maccione, Günter Sigl and Le Zhang for helpful discussions. A.I. would like to thank the Korea Institute for Advanced Study (KIAS) for hospitality during the last stages of this work. The work of A.I. and D.T. was partially supported by the DFG cluster of excellence "Origin and Structure of the Universe."

\section{APPENDIX A: STATISTICAL ERRORS OF THE LARGE-SCALE ANISOTROPY}

Here, we shortly discuss the statistical errors of the large-scale anisotropy as defined in Eq. (12), which are due to shot noise. Statistical errors of small-scale anisotropies are discussed e.g. in Ref. 78.

The measured anisotropy $A$ and the total number of measured photons $N$ are related to the number of photons measured in direction of the Galactic center, $N_{1}$, and anticenter, $N_{2}$, by $A=\left(N_{1}-N_{2}\right) /\left(N_{1}+N_{2}\right)$ and $N=N_{1}+N_{2}$. The $N_{i}$ follow a Poisson distribution with mean $\left\langle N_{i}\right\rangle$ and standard deviation $\sigma_{N_{i}}=\sqrt{\left\langle N_{i}\right\rangle}$. Considering the propagation of uncertainty, it is straightforward to derive that the statistical error of the anisotropy is given by

$$
\sigma_{A} \simeq \sqrt{\frac{1-\langle A\rangle^{2}}{\langle N\rangle}}
$$

which is expected to hold for small enough $\langle A\rangle \simeq A$ and large enough $\langle N\rangle \simeq N$.

On the other hand, one can derive the exact probability distribution function of the anisotropy $A$ by starting with the above Poisson distributions for the $N_{i}$, performing an appropriate redefinition of the parameters and integrating out the total number of measured photons. The result is a function of the mean values $\langle A\rangle$ and $\langle N\rangle$ and can be written in the compact form

$$
p d f(A)=\frac{\langle N\rangle}{2\left\langle N_{1}\right\rangle !\left\langle N_{2}\right\rangle !}\left(\frac{1+A}{2}\right)^{\left\langle N_{1}\right\rangle}\left(\frac{1-A}{2}\right)^{\left\langle N_{2}\right\rangle} .
$$

From this equation one can check, for example, that a normal distribution with mean $\langle A\rangle$ and standard deviation as in Eq. (A1) gives correct error bars at the $5 \%$ level as long as $\langle A\rangle<0.6$ and $\sigma_{A}<0.2$. For small enough anisotropies $A$, however, the standard deviation is just given by $\sigma_{A}=\sqrt{N^{-1}}$ with good accuracy, and we use this approximation throughout the work.

\section{APPENDIX B: OBSERVATION OF PROMPT AND ICS RADIATION FROM DARK MATTER DECAY}

In this appendix we will briefly discuss the differences between observing ICS radiation from positrons and electrons from dark matter decay and observing the gamma rays that come directly from the decay itself (prompt radiation like internal bremsstrahlung) by means of signalto-noise and signal-to-background ratios.

Signal-to-noise ratios quantify the significance of a signal against statistical noise. The signal-to-noise ratio $S / N$ of a dark matter signal with respect to the background is given by

$$
\frac{S}{N}=\frac{N_{\gamma, \mathrm{s}}}{\sqrt{N_{\gamma, \mathrm{s}}+N_{\gamma, \mathrm{bg}}}},
$$

where $N_{\gamma, \mathrm{s}}$ and $N_{\gamma, \text { bg }}$ denote the number of detected signal and background photons, respectively, that are observed in a given sky region $\Delta \Omega$ and energy band $E_{\gamma}=E_{0} \ldots E_{1}$. Signal photons are all photons from dark matter decay, background photons are in principle all other observed photons, including the astrophysical part of the EGBG and the Galactic foreground. Since the number of detected photons scales like $N_{\gamma, i} \propto$ $\int_{\Delta \Omega} d \Omega \int_{E_{0}}^{E_{1}} d E d J_{i} / d E_{\gamma} \equiv \Delta \Omega \bar{J}_{i}$, the signal-to-noise ratio in the limit $\Delta \Omega \rightarrow 0$ is proportional to

$$
\frac{S}{N} \propto \frac{\bar{J}_{\mathrm{s}}}{\sqrt{\bar{J}_{\mathrm{s}}+\bar{J}_{\mathrm{bg}}}}
$$

where $\bar{J}_{\mathrm{s}}$ and $\bar{J}_{\mathrm{bg}}$ denote the appropriately averaged and integrated signal and background gamma-ray fluxes, respectively.

In the upper panel of Fig. 8 we plot the relative signalto-noise ratio (with arbitrary normalization), assuming that the background completely dominates the signal, $\bar{J}_{\mathrm{s}} \ll \bar{J}_{\mathrm{bg}}$, and neglecting ICS and extragalactic radiation. As background we take the predictions of the conventional GALPROP model at $E_{\gamma}=100 \mathrm{GeV}$ (from Ref. 63], see above), but the results do not change qualitatively for other energies. The anisotropy of the dark matter signal as function of $l$ is well recognizable in the plot. Furthermore it is apparent that, from the perspective of statistical noise, the sky regions that are most sensitive to decaying dark matter signals lie close above and below the Galactic center, with $|l| \lesssim 25^{\circ}$ and $5^{\circ} \lesssim|b| \lesssim 35^{\circ}$.

To determine the best observational strategy in light of the systematics that are related to the determination of the Galactic foreground, it is more convenient to consider the signal-to-background ratio $J_{\mathrm{s}} / J_{\mathrm{bg}}$ (we assume 

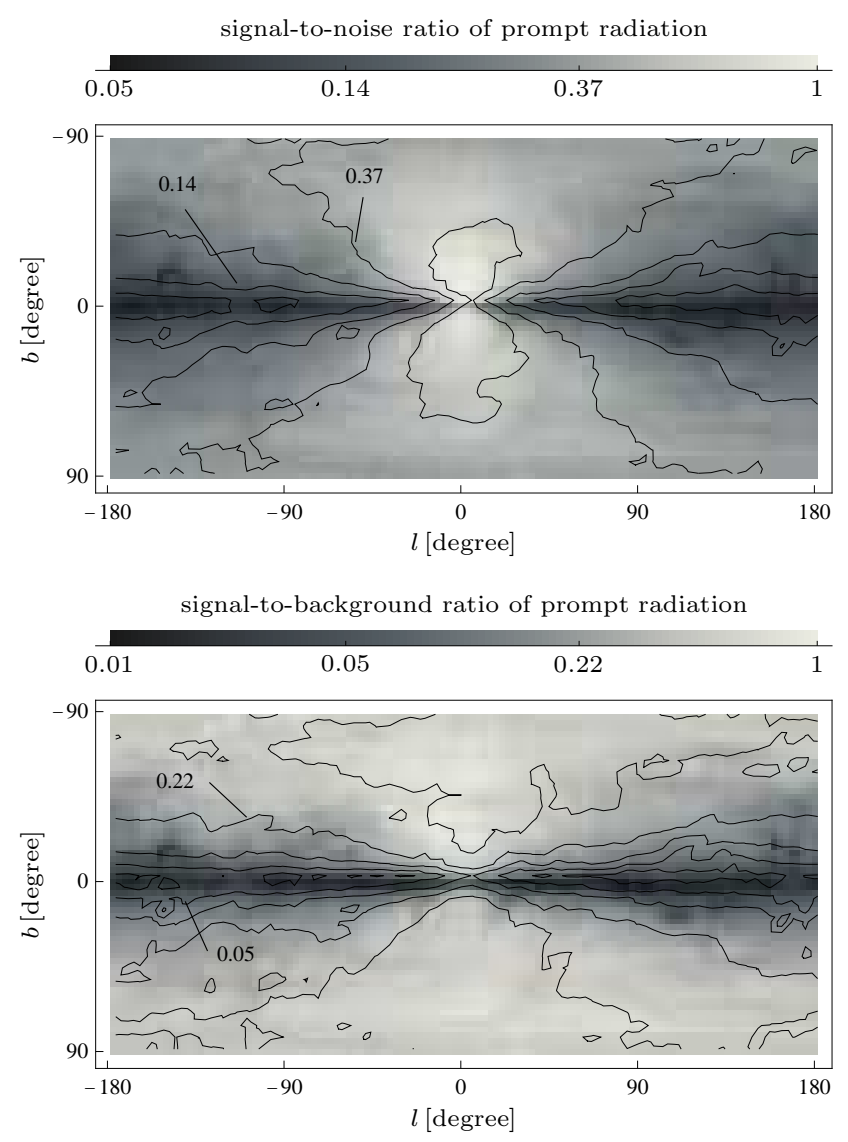

FIG. 8: Upper panel: Relative signal-to-noise ratio of gammaray signal from dark matter decay as a function of the Galactic longitude $l$ and latitude $b$, normalized to one at the Galactic center. Lower panel: Relative signal-to-background ratio for the same process. Extragalactic and ICS radiation is neglected, and as background we take the predictions of the conventional Galprop model at $100 \mathrm{GeV}$.

that systematic uncertainties scale roughly like $\sim J_{\mathrm{bg}}$ ). We show the signal-to-background ratio as a function of the Galactic coordinates in the lower panel of Fig. 8 , Again, the large-scale anisotropy of the dark matter flux are clearly visible in the plot. Furthermore, it is apparent that concerning systematics the best strategy is to avoid regions near the Galactic plane and to observe fluxes only at higher latitudes, $|b| \gtrsim 20^{\circ}$. However, as a compromise between statistical and systematic uncertainties we choose to consider the whole region $10^{\circ} \leq|b| \leq 90^{\circ}$ in most of the present paper.

In contrast to gamma rays that come directly from the dark matter decay, the gamma rays that stem from inverse Compton scattering of dark matter positrons or electrons on the ISRF are mostly coming from the region near the Galactic center. This can be seen in Fig. 9 , where we plot the signal-to-background ratio of the pure ICS signal of dark matter decaying into an $e^{+} e^{-}$pair (with $m_{\mathrm{dm}}=1 \mathrm{TeV}$ ) as a function of the Galactic co-

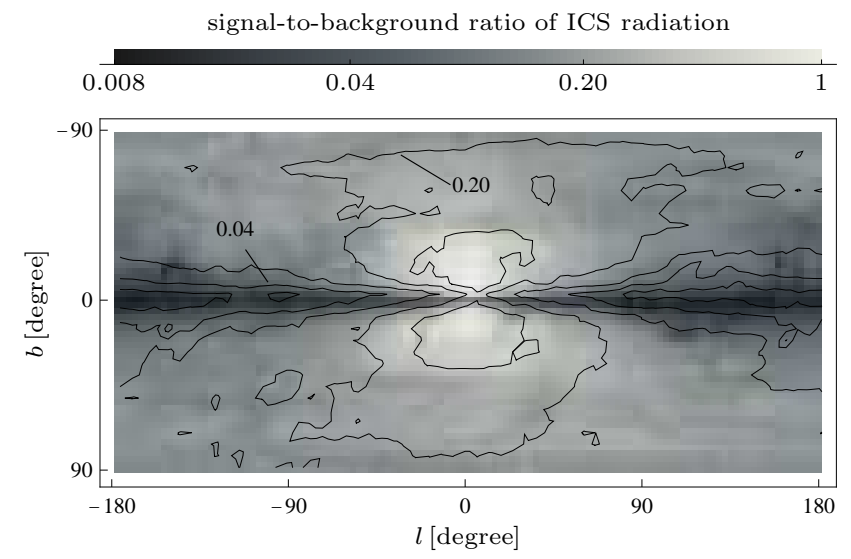

FIG. 9: Relative signal-to-background ratio of pure ICS radiation from dark matter decay at $E_{\gamma}=10 \mathrm{GeV}$. We assume that $m_{\mathrm{dm}}=1 \mathrm{TeV}$ and that the dark matter is decaying into $e^{+} e^{-}$pairs. As background we take the fluxes from the conventional GALPROP model.

ordinates. The gamma-ray energy is $E_{\gamma}=10 \mathrm{GeV}$ in this plot. As background we again use the predictions from the conventional GALPROP model. From the figure it is apparent that the relative size of the signal peaks at regions very close below and above the Galactic center, with $|l| \lesssim 20^{\circ}$ and $5^{\circ} \lesssim|b| \lesssim 30^{\circ}$. This suggests that concentrating the observation on these regions is most promising for the search for ICS radiation from dark matter decay. However, in light of the large underlying uncertainties related to the predictions of ICS radiation we will neglect these subtleties, and we consider ICS radiation only in how far it affects the anisotropies and fluxes in the sky regions that are most promising for the search of gamma rays coming from the dark matter decay itself.
[1] P. L. Nolan et al., Astrophys. J. 459, 100 (1996), astro$\mathrm{ph} / 9509023$.

[2] D. Grasso et al. (2009), 0905.0636.

[3] O. Adriani et al. (PAMELA), Nature 458, 607 (2009), 0810.4995.

[4] A. A. Abdo et al. (The Fermi LAT), Phys. Rev. Lett. 102, 181101 (2009), 0905.0025.
[5] S. W. Barwick et al. (HEAT), Astrophys. J. 482, L191 (1997), astro-ph/9703192.

[6] C. Grimani, Astron. Astrophys. 418, 649 (2004); A. K. Harding and R. Ramaty, Proc. 20th ICRC, Moscow 2, 92 (1987); A. M. Atoian, F. A. Aharonian, and H. J. Volk, Phys. Rev. D52, 3265 (1995); X. Chi, E. C. M. Young, and K. S. Cheng, Astrophys. J. 459, L83 (1995). 
[7] D. Hooper, P. Blasi, and P. D. Serpico, JCAP 0901 , 025 (2009), 0810.1527; H. Yuksel, M. D. Kistler, and T. Stanev, Phys. Rev. Lett. 103, 051101 (2009), 0810.2784 .

[8] D. Malyshev, I. Cholis, and J. Gelfand (2009), 0903.1310.

[9] S. Profumo (2008), 0812.4457.

[10] L. Bergstrom, J. Edsjo, and G. Zaharijas, Phys. Rev. Lett. 103, 031103 (2009), 0905.0333.

[11] P. Meade, M. Papucci, A. Strumia, and T. Volansky (2009), 0905.0480

[12] X.-J. Bi et al. (2009), 0905.1253.

[13] F. Chen, J. M. Cline, and A. R. Frey (2009), 0907.4746.

[14] A. Ibarra, A. Ringwald, D. Tran, and C. Weniger, JCAP 0908, 017 (2009), 0903.3625.

[15] A. Ibarra, D. Tran, and C. Weniger (2009), 0906.1571.

[16] A. Ibarra and D. Tran, JCAP 0807, 002 (2008), 0804.4596; A. Ibarra and D. Tran, Phys. Rev. Lett. 100, 061301 (2008), 0709.4593; K. Ishiwata, S. Matsumoto, and T. Moroi, Phys. Rev. D78, 063505 (2008), 0805.1133; W. Buchmuller, A. Ibarra, T. Shindou, F. Takayama, and D. Tran (2009), 0906.1187; L. Covi, M. Grefe, A. Ibarra, and D. Tran, JCAP 0901, 029 (2009), 0809.5030.

[17] C.-R. Chen, F. Takahashi, and T. T. Yanagida, Phys. Lett. B671, 71 (2009), 0809.0792; C.-R. Chen and F. Takahashi, JCAP 0902, 004 (2009), 0810.4110; P.-f. Yin et al., Phys. Rev. D79, 023512 (2009), 0811.0176; K. Hamaguchi, S. Shirai, and T. T. Yanagida, Phys. Lett. B673, 247 (2009), 0812.2374; M. Pospelov and M. Trott, JHEP 04, 044 (2009), 0812.0432; S. Shirai, F. Takahashi, and T. T. Yanagida (2009), 0902.4770; A. Arvanitaki et al. (2009), 0904.2789; K. J. Bae and B. Kyae, JHEP 05, 102 (2009), 0902.3578; S.-L. Chen, R. N. Mohapatra, S. Nussinov, and Y. Zhang, Phys. Lett. B677, 311 (2009), 0903.2562; H. Fukuoka, J. Kubo, and D. Suematsu, Phys. Lett. B678, 401 (2009), 0905.2847; J. Hisano, K. Nakayama, and M. J. S. Yang, Phys. Lett. B678, 101 (2009), 0905.2075; A. Arvanitaki et al., Phys. Rev. D79, 105022 (2009), 0812.2075.

[18] K. Kohri, A. Mazumdar, N. Sahu, and P. Stephens (2009), 0907.0622; J. Mardon, Y. Nomura, and J. Thaler, Phys. Rev. D80, 035013 (2009), 0905.3749; N. Okada and T. Yamada (2009), 0905.2801; S. Shirai, F. Takahashi, and T. T. Yanagida (2009), 0905.0388; C.-H. Chen, C.-Q. Geng, and D. V. Zhuridov (2009), 0906.1646; C.-H. Chen, C.-Q. Geng, and D. V. Zhuridov (2009), 0905.0652

[19] J. Dunkley et al. (WMAP), Astrophys. J. Suppl. 180, 306 (2009), 0803.0586.

[20] G. Bertone, D. Hooper, and J. Silk, Phys. Rept. 405, 279 (2005), hep-ph/0404175.

[21] P. Ullio, L. Bergstrom, J. Edsjo, and C. G. Lacey, Phys. Rev. D66, 123502 (2002), astro-ph/0207125.

[22] L. Pieri, J. Lavalle, G. Bertone, and E. Branchini (2009), 0908.0195.

[23] M. Regis and P. Ullio, Phys. Rev. D80, 043525 (2009), 0904.4645.

[24] A. Ibarra, A. Ringwald, and C. Weniger, JCAP 0901, 003 (2008), 0809.3196.

[25] W. Buchmuller, L. Covi, K. Hamaguchi, A. Ibarra, and T. Yanagida, JHEP 03, 037 (2007), hep-ph/0702184.

[26] M. R. Buckley, K. Freese, D. Hooper, D. Spolyar, and H. Murayama (2009), 0907.2385.

[27] A. Ibarra and D. Tran, JCAP 0906, 004 (2009),
0904.1410.

[28] M. Kadastik, M. Raidal, and A. Strumia (2009), 0908.1578.

[29] D. Spolyar, M. Buckley, K. Freese, D. Hooper, and H. Murayama (2009), 0905.4764.

[30] K. Ishiwata, S. Matsumoto, and T. Moroi, Phys. Lett. B675, 446 (2009), 0811.0250.

[31] A. Ibarra and D. Tran, JCAP 0902, 021 (2009), 0811.1555 .

[32] J. T. Ruderman and T. Volansky (2009), 0907.4373.

[33] G. Bertone, W. Buchmuller, L. Covi, and A. Ibarra, JCAP 0711, 003 (2007), 0709.2299.

[34] S. Gillessen et al., Astrophys. J. 692, 1075 (2009), 0810.4674

[35] V. Springel et al., Mon. Not. Roy. Astron. Soc. 391, 1685 (2008), 0809.0898.

[36] J. F. Navarro et al., Mon. Not. Roy. Astron. Soc. 349, 1039 (2004), astro-ph/0311231.

[37] R. Catena and P. Ullio (2009), 0907.0018.

[38] M. Cirelli and P. Panci, Nucl. Phys. B821, 399 (2009), 0904.3830.

[39] K. Ishiwata, S. Matsumoto, and T. Moroi, Phys. Lett. B679, 1 (2009), 0905.4593.

[40] M. D. Kistler and J. M. Siegal-Gaskins (2009), 0909.0519.

[41] S. Profumo and T. E. Jeltema, JCAP 0907, 020 (2009), 0906.0001

[42] J. Zhang et al., Phys. Rev. D80, 023007 (2009), 0812.0522

[43] E. Borriello, A. Cuoco, and G. Miele, Astrophys. J. 699, L59 (2009), 0903.1852.

[44] G. R. Blumenthal and R. J. Gould, Rev. Mod. Phys. 42, 237 (1970).

[45] L. Zhang, G. Sigl, and J. Redondo (2009), 0905.4952.

[46] K. Ishiwata, S. Matsumoto, and T. Moroi, Phys. Rev. D79, 043527 (2009), 0811.4492.

[47] I. V. Moskalenko and A. W. Strong, Astrophys. J. 528, 357 (2000), astro-ph/9811284.

[48] T. A. Porter and A. W. Strong (2005), astro-ph/0507119.

[49] I. V. Moskalenko and A. W. Strong, Astrophys. J. 493, 694 (1998), astro-ph/9710124.

[50] A. W. Strong, I. V. Moskalenko, and V. S. Ptuskin, Ann. Rev. Nucl. Part. Sci. 57, 285 (2007), astro-ph/0701517.

[51] T. Delahaye, R. Lineros, F. Donato, N. Fornengo, and P. Salati, Phys. Rev. D77, 063527 (2008), 0712.2312.

[52] R. J. Gould and G. P. Schreder, Phys. Rev. 155, 1404 (1967).

[53] F. W. Stecker, M. A. Malkan, and S. T. Scully, Astrophys. J. 648, 774 (2006), astro-ph/0510449; see also erratum F. W. Stecker, M. A. Malkan, and S. T. Scully, Astrophys. J. 658, 1392 (2007), astro-ph/0612048.

[54] A. Cuoco, J. Brandbyge, S. Hannestad, T. Haugboelle, and G. Miele, Phys. Rev. D77, 123518 (2008), 0710.4136.

[55] I. V. Moskalenko, T. A. Porter, and A. W. Strong, Astrophys. J. 640, L155 (2006), astro-ph/0511149.

[56] C. D. Dermer, AIP Conf. Proc. 921, 122 (2007), 0704.2888.

[57] P. Sreekumar et al. (EGRET), Astrophys. J. 494, 523 (1998), astro-ph/9709257.

[58] A. W. Strong, I. V. Moskalenko, and O. Reimer, Astrophys. J. 613, 956 (2004), astro-ph/0405441.

[59] D. Hooper and P. D. Serpico, JCAP 0706, 013 (2007), astro-ph/0702328.

[60] C. Calcaneo-Roldan and B. Moore, Phys. Rev. D62, 123005 (2000), astro-ph/0010056. 
[61] A. N. Cillis and R. C. Hartman, Astrophys. J. 621, 291 (2005).

[62] I. V. Moskalenko and T. A. Porter, Astrophys. J. 692, 54 (2009), 0901.0304.

[63] A. W. Strong, I. V. Moskalenko, and O. Reimer, Astrophys. J. 613, 962 (2004), astro-ph/0406254.

[64] M. Ackermann, Talk given at TeV Particle Astrophysics 2009, URL www-conf.slac.stanford.edu/tevpa09/

[65] T. Sjostrand, S. Mrenna, and P. Skands, JHEP 05, 026 (2006), hep-ph/0603175.

[66] V. Barger, Y. Gao, W. Y. Keung, and D. Marfatia (2009), 0906.3009.

[67] E. A. Baltz et al., JCAP 0807, 013 (2008), 0806.2911.

[68] S. Ando and E. Komatsu, Phys. Rev. D73, 023521 (2006), astro-ph/0512217.

[69] S. Ando, E. Komatsu, T. Narumoto, and T. Totani, Phys. Rev. D75, 063519 (2007), astro-ph/0612467.

[70] S. Ando, Phys. Rev. D80, 023520 (2009), 0903.4685.
[71] M. Fornasa, L. Pieri, G. Bertone, and E. Branchini, Phys. Rev. D80, 023518 (2009), 0901.2921.

[72] J. M. Siegal-Gaskins, JCAP 0810, 040 (2008), 0807.1328.

[73] J. M. Siegal-Gaskins and V. Pavlidou, Phys. Rev. Lett. 102, 241301 (2009), 0901.3776.

[74] L. Pieri, G. Bertone, and E. Branchini, Mon. Not. Roy. Astron. Soc. 384, 1627 (2008), 0706.2101.

[75] A. Cooray and R. K. Sheth, Phys. Rept. 372, 1 (2002), astro-ph/0206508.

[76] S. Ando, E. Komatsu, T. Narumoto, and T. Totani, Mon. Not. Roy. Astron. Soc. 376, 1635 (2007), astro$\mathrm{ph} / 0610155$.

[77] F. Miniati, S. M. Koushiappas, and T. Di Matteo, Astrophys. J. 667, L1 (2007), astro-ph/0702083.

[78] A. Cuoco et al., JCAP 0704, 013 (2007), astro$\mathrm{ph} / 0612559$. 